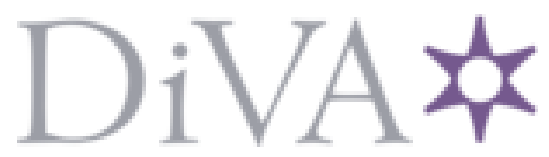

http://www.diva-portal.org

\title{
Postprint
}

This is the accepted version of a paper presented at CHI '13 Extended Abstracts on Human Factors in Computing Systems.

Citation for the original published paper:

Vines, J., McNaney, R., Clarke, R., Lindsay, S., McCarthy, J. et al. (2013)

Designing For- and With- Vulnerable People.

In: CHI '13 Extended Abstracts on Human Factors in Computing Systems (pp. 3231-3234).

CHI EA '13

http://dx.doi.org/10.1145/2468356.2479654

N.B. When citing this work, cite the original published paper.

Permanent link to this version:

http://urn.kb.se/resolve?urn=urn:nbn:se:kth:diva-184684 


\section{Designing For- and With- Vulnerable People}

\section{John Vines}

Culture Lab,

School of Computing Science,

Newcastle University, UK

john.vines@ncl.ac.uk

\section{Roisin McNaney}

Culture Lab,

School of Computing Science,

Newcastle University, UK

r.mcnaney@ncl.ac.uk

\section{Rachel Clarke}

Culture Lab,

Newcastle University, UK

r.clarke@ncl.ac.uk

\section{Stephen Lindsay}

Dept. of Computer Science

Swansea University, UK

s.c.lindsay@swansea.ac.uk
School of Computing Science,

\section{John McCarthy}

School of Applied Psychology,

University College Cork, Ireland

john.mccarthy@ucc.ie

\section{Steve Howard}

Dept. of Computing \& Information

Systems,

The University of Melbourne,

showard@unimelb.edu.au

\section{Mario Romero}

Dept. of Mathematics,

Uppsala Universitet,

mario.romero@math.uu.se

\section{Jayne Wallace}

School of Design,

Northumbria University, UK

jayne.wallace@northumbria.ac.uk

Copyright is held by the author/owner(s).

CHI 2013 Extended Abstracts, April 27-May 2, 2013, Paris, France.

ACM 978-1-4503-1952-2/13/04.

\begin{abstract}
Increasingly ubiquitous technology coupled with a surge in empirical research on people who face cognitive, emotional, physical and social challenges increases the potential for HCI to enrich the lives of vulnerable people. Designing for people with vulnerabilities requires an approach to participation that is sensitive to the risks of possible stigmatization and an awareness of the challenges for participant involvement. This workshop will bring together researchers and practitioners to explore the critical issues surrounding designing with and for vulnerable individuals. We aim to provoke discussion about how 'vulnerability' is defined in $\mathrm{HCI}$, what methodological and ethical concerns are raised when working with specific cases, and ways of designing for future technologies that support vulnerable people in novel and sensitive ways.
\end{abstract}

\section{Author Keywords}

Design; vulnerable individuals; methods; ethics.

\section{ACM Classification Keywords}

H.5.m [Information interfaces and presentation (e.g., $\mathrm{HCI})]$ : Miscellaneous;

\section{Introduction}

People can be vulnerable in a variety of ways, physically, cognitively, emotionally, and socially [3]. In many cases these vulnerabilities interact with each other. So, for example, a person with dementia may 
face emotional and social, as well as the more apparent cognitive difficulties. This variety of vulnerabilities, and the likelihood of their interaction in people's lived experience, presents real challenges for HCI design. Research in health and social sciences on people who live with these challenges has grown significantly in recent years $[1,7,11,13]$, while others have critically examined how vulnerabilities are often socially constructed and can be accentuated by technology [8]. This suggests great potential for significant contributions to improving quality of life from accessible and assistive technologies to experience-centered design interventions.

Much prior HCI work has examined the experiences and needs of people who might be considered vulnerable. This has included working with groups as diverse as homeless mothers [5], people living with dementia [6], children with special needs [4] and parolees and parole officers [11] among many others. It has also lead to dealing with highly sensitive topics that potentially place participants in a vulnerable position, such as recollections of domestic abuse [1], and topics related to death and the end of life [7]. Work with individuals perceived to be vulnerable can also lead to great design innovations, such as the development of new tools to support visually impaired people to communicate [8], information exchange between homeless people [5] and social support tools for breast cancer patients [11]

This workshop aims to synthesize and build upon this prior work by bringing together a diverse community to share existing knowledge, define new research and design opportunities and outline an agenda for future research on technology with and for vulnerable groups.

\section{Topics and Goals}

We aim to consider the scope of issues that arise when working with vulnerable groups with the goal of developing a design- and conceptual- framework to support and shape future research. We will address this challenge through three themes in the workshop:

\section{Examples of vulnerability}

The workshop will bring together participants from multiple research areas surrounding HCI to discuss and define the factors that they consider make people vulnerable. In attempting to define vulnerability, we ask: What do we associate with the word vulnerable? How do we identify groups and individuals as 'vulnerable'? What effects can this classification have on the individual? Are medical and legal definitions of vulnerability used in HCI research, and are these useful or problematic? How do we go about defining vulnerability when we consider the range of groups associated with the term? Finally, we ask what effects do being classified as 'vulnerable' have on the individual and whether the term is a pejorative one?

Researching vulnerability

In discussing examples of vulnerability within $\mathrm{HCI}$ we will be better placed to explore what impact working with vulnerable individuals and groups has on research processes-and, vis-à-vis, what impact the research process has on those who participate. Here, we ask: By working with or designing for particular groups do we as researchers make them vulnerable? Are there contexts where researchers themselves become vulnerable due to the topic being explored or the people they are working with? How might researchers be actively contributing to the defining of a group as 'vulnerable' simply by working with them? What are the 
ethical and moral issues in this space, and is it necessary to explore less intrusive, novel approaches to understanding and working with these groups?

Furthermore, are there generalisable practical challenges to the collection of data when working with vulnerable groups?

Designing for and with vulnerable individuals By placing an emphasis on definitions, research and ethical issues related to vulnerability, we aim to provoke new ideas about how to approach the design of new technologies to support such individuals in the future. By questioning how the $\mathrm{HCI}$ community typically defines people as vulnerable (or not) we hope to inspire alternative visions of technology where individuals are provided means to having a voice rather than being stigmatized as vulnerable or in need of assistance.

\section{Intended audience}

We propose a one-day workshop, to be attended by the authors of 15-20 accepted submissions. The workshop intends to support multi-disciplinary discussion around technology design for vulnerable groups, using technology to support working with such groups, and specific issues and considerations that must be made when undertaking HCI research with these individuals. We will consider the scope of issues that might arise when working with vulnerable groups by asking workshop participants to draw on their individual experiences to carefully dissect and discuss a range of case studies relating to the design of digital technologies with specified vulnerable groups. We invite the submission of 2-4 page position papers offering perspectives on these topics. We will solicit submissions from researchers, designers and practitioners involved in fields such as healthcare, social sciences and interaction design.

\section{Deadlines}

$11^{\mathrm{h}}$ Jan 2013: Workshop submission deadline.

$8^{\text {th }}$ Feb 2013: Feedback and notifications to authors. $27^{\text {th }}$ or $28^{\text {th }}$ Apr 2013: Workshop at CHI 2013.

\section{Workshop Format}

The workshop will be scheduled as follows:

09:00- 09:15 Welcome and introductions

09:15- 10:30 Participant plenary introductions

10:30-11:00 Coffee break

11:00-12:30 Examples of vulnerability (Group work)

12:30- 13:00 Identifying shared themes

13:00- 14:00 Workshop lunch

14:00- 15:30 Focused group work on themes

15:30- 16:00 Coffee break

16:00- 17:30 Practical group activity, discussion of group work and wrap-up

The workshop will be a balance between briefpresentations, focused group discussions and practical design activities. The 'madness' session will provide valuable insights about individual perspectives on vulnerability and experiences designing with these groups. Next, workshop participants will attempt to define vulnerability through breakout sessions focusing on specific issues, e.g. What do we associate with the word vulnerable? How do we go about identifying groups and individuals as 'vulnerable'? What effects can this classification have on the individual?

During and after lunch participants will be asked to identify themes for the next session. The group will be asked to come up with a series of case studies with a focus on technology design with or for a vulnerable 
user group (structured as: target user group, why they are identified as vulnerable, what are we designing to help the user? How can we use technology to support working with this user group, if at all? What are the methodological considerations when working with this group? What are the design considerations that must be applied to the end technology?). The workshop organisers will have a set of these case studies preprepared to facilitate group discussion and the flow of ideas and will provide materials to assist the task. Teams will report on their work, considering issues including user groups, design rationale, ethical considerations and potential benefit and pitfalls of their designs. The workshop will conclude with an open discussion to consolidate ideas generated throughout the day and establish a future agenda.

\section{Dissemination}

During the workshop we will prepare material for a poster for the $\mathrm{CHI}$ spotlight on workshops session. Following this, we will write an article on the workshop for a SIGCHI members publication (e.g. Interactions) and a report on findings will be made available openly online. Furthermore, a special issue journal submission on Designing for Vulnerable Groups will be proposed and a conceptual 'toolkit' to support researchers working and collaborating with vulnerable people and groups will be an outcome of the workshop. After the workshop we will update the webpage to reflect the workshop outcomes and make materials available to the $\mathrm{CHI}$ community. We will continue to use the homepage (http://di.ncl.ac.uk/vulnerability) as a basis for discussion and community building activities.

\section{References}

[1] Clare, L. et al. The experience of living with dementia in residential care: An interpretative phenomenological approach. (2008). The Gerontologist, 48 (6), 711-720

[2] Clarke, R., and Wright, P. Evocations of experience: Crafting cross-cultural digital narratives through stories and portraits. In: Proc. NordiCHI '12.

[3] Department of Health. (2000) No Secrets. DoH, UK

[4] Frauenberger, C., Good, J., and Keay-Bright, W. Interpreting input from children: A designerly approach. In: Proc. CHI '12, 2377-2386.

[5] Le Dantec, C. et al. Publics in practice: Ubiquitous computing at a shelter for homeless mothers. In: Proc. CHI'11, 1687-1696.

[6] Lindsay, S., Brittain, K. et al. Empathy, participatory design and people with dementia. In: Proc. CHI '12, 521-530.

[7] Massimi, M., Odom, W., Banks, R., and Kirk, D. Matters of life and death Locating the end of life in lifespan-oriented HCI research. In: Proc. CHI '11, 987996.

[8] Oliver, M. Understanding disability: From theory to practice. Macmillian, Basingstoke, 1996.

[9] Romero, M., Frey, B., Souther, C., and Abowd, G. BrailleTouch: Designing a mobile eyes-free soft keyboard. In: Proc. MobileHCI '11, 707-709.

[10] Schneider, K. To the Left of Inspiration: Adventures in living with disabilities. Dog Ear Publishing, IN, USA

[11] Scklovski, I., et al. The commodification of location: Dynamics of power in location-based systems. In: Proc. UbiComp '09, 11-20.

[12] Skeels, M., et al. Catalyzing social support for breast cancer patients. In: Proc. CHI '10, 173-182.

[13] Wallace, J., Thieme, A., Wood, G., Schofield, G., and Olivier, P. Enabling self, intimacy and a sense of home in dementia: An enquiry into design in a hospital setting. In: Proc. CHI '12, 2629-2638. 